\title{
Full-diversity STBCs for Block-Fading channels from Cyclic codes
}

\author{
U. Sripati \\ ECE Department \\ Indian Institute of Science \\ Bangalore - 560012 INDIA \\ Email: sripati@protocol.ece.iisc.ernet.in
}

\author{
B. Sundar Rajan \\ ECE Department \\ Indian Institute of Science \\ Bangalore - 560012 INDIA \\ Email: bsrajan@ece.iisc.ernet.in
}

\author{
Shashidhar V \\ ECE Department \\ Indian Institute of Science \\ Bangalore - 560012 INDIA \\ Email: shashidhar@protocol.ece.iisc.ernet.in
}

\begin{abstract}
Viewing an $n$-length vector over $F_{q}$ (the finite field of $q^{m}$ elements) as an $m \times n$ matrix over $F_{q}$, by expanding each entry of the vector with respect to a basis of $F_{q} m$ over $F_{q}$, the rank weight of the $n$-length vector over $F_{q^{m}}$ is the rank of the corresponding $m \times n$ matrix over $F_{q}$. Using appropriate Discrete Fourier Transform (DFT), it is known that under some conditions, $n$-length cyclic codes over $F_{q^{m}},\left(n \mid q^{m}-1\right.$ and $\left.m \leq n\right)$, have full-rank $(=m)$. In this paper, using this result, we obtain designs for Full-diversity Space Time Block Codes (STBCs) suitable for block-fading channels from $n$ length cyclic codes over $F_{q}$. These STBCs are suitable for $m$ transmit antennas over signal sets matched to $F_{q}$, where $q=2$ or $q$ is a prime of the form $4 k+1,(k=1,2, \cdots)$. We also present simulation results which illustrate the performance of a few of these STBCs and show that our codes perform better than the well known codes for block-fading channels.
\end{abstract}

\section{INTRODUCTION}

An $m \times l(m \leq l)$ Space Time Block Code (STBC) $\mathcal{C}$ for $m$ transmit antennas over a complex signal set $S$ is a finite number of $m \times l$ matrices with entries from $S$. Let us consider a system with $N_{t}$ transmit antennas, $N_{r}$ receive antennas and codeword length $l$. In the block-fading model, the codeword is considered to be composed of multiple blocks. The channel fading coefficients are constant over each block and are independent from block to block. For an STBC for a block-fading channel, $l$ is an integral multiple of the length of the block. Let $M$ denote the number of blocks in a codeword. Then, the size of each block is $\delta \triangleq \frac{l}{M}$. When, the codeword length extends over several quasi-static blocks, as in the case of block-fading channels, El Gamal and Hammons [1] have shown that the diversity can be made to increase indefinitely with increase in length of the codeword. In [2], it was shown that the limiting performance in terms of codeword error rate can be improved by coding across multiple blocks. In particular, it was shown that the negative of the exponent of SNR in the codeword error rate for a fixed data rate can be increased to any value by coding across multiple blocks. The following base-band design criteria for block-fading channels have been derived in [1].

Design Criteria for block-fading channels [1]: Let $\mathcal{C}$ be an $n \times l$ STBC code where $\mathbf{c}^{\prime}=\left[c^{\prime}[1], c^{\prime}[2], \cdots c^{\prime}[M]\right]$ and

\footnotetext{
${ }^{1}$ This work was partly funded by the IISc-DRDO programme on Advanced Research in Mathematical Engineering through a grant to B.S.Rajan.
}

$\mathbf{e}^{\prime}=\left[e^{\prime}[1], e^{\prime}[2] \cdots e^{\prime}[M]\right]$ are any two distinct codewords and $\mathbf{c}^{\prime}[\tau], \mathbf{e}^{\prime}[\tau], 1 \leq \tau \leq M$ are the $\tau^{t h}$ blocks of codewords $\mathbf{c}^{\prime}, \mathbf{e}^{\prime}$ respectively. The pairwise probability of error is

$$
P\left(\mathbf{c}^{\prime} \rightarrow \mathbf{e}^{\prime}\right) \leq \prod_{\tau=1}^{M}\left(\frac{\mu_{\tau} E_{s}}{4 N_{0}}\right)^{-d_{\tau} N_{r}}
$$

where

$$
\begin{gathered}
\mu_{\tau}=\left(\lambda_{1}[\tau] \lambda_{2}[\tau] \cdots \lambda_{d_{\tau}}[\tau]\right)^{\frac{1}{d_{\tau}}}, \\
d_{\tau}=\operatorname{rank}\left(c^{\prime}[\tau]-e^{\prime}[\tau]\right)
\end{gathered}
$$

and $\lambda_{1}[\tau], \lambda_{2}[\tau], \cdots \lambda_{d_{\tau}}[\tau]$ are the non zero eigen values of $\mathbf{A}[\tau]=\left(\mathbf{c}^{\prime}[\tau]-\mathbf{e}^{\prime}[\tau]\right)\left(\mathbf{c}^{\prime}[\tau]-\mathbf{e}^{\prime}[\tau]\right)^{H}$, Hence the generalized diversity and product distance criteria for STBCs over MIMO block-fading channels can be stated as follows :

(i) Block-fading sum of ranks criterion : Maximize the transmit diversity advantage,

$$
d=\sum_{\tau=1}^{M} d_{\tau}=\sum_{\tau=1}^{M} \operatorname{rank}\left(\mathbf{c}^{\prime}[\tau]-\mathbf{e}^{\prime}[\tau]\right)
$$

over all pairs of distinct codewords $\mathbf{c}^{\prime}, \mathbf{e}^{\prime} \in \mathcal{C}$.

From this criterion, it follows that the diversity advantage increases rapidly with increase in the number of blocks $M$ in the codeword if, for all pairwise differences of codewords, the difference blocks $\left(\mathbf{c}^{\prime}(\tau)-\mathbf{e}^{\prime}(\tau)\right), 1 \leq \tau \leq M$ have fullrank.

(ii) Block-fading Product distance criterion : Maximise the coding advantage,

$$
\prod_{\tau=1}^{M} \mu_{\tau}=\prod_{\tau=1}^{M}\left\{\left(\lambda_{1}[\tau] \lambda_{2}[\tau] \cdots \lambda_{d_{\tau}}[\tau]\right)^{\frac{1}{d_{\tau}}}\right\}
$$

over all pairs of distinct codewords $\mathbf{c}^{\prime}, \mathbf{e}^{\prime} \in \mathcal{C}$.

In [1], full-diversity codes over BPSK and QPSK signal sets have been constructed. In these codes, the coding is done across 2, 3 and 4 blocks. In [3], full-diversity codes with coding across 2, 3 and 4 blocks were constructed for QPSK signal set.

Let $\mathbf{C}$ be an $[\mathrm{n}, \mathrm{k}]$ linear code over $F_{q^{m}}$. For any pair of codewords, $\mathbf{c}, \mathbf{e} \in \mathbf{C}$, the rank distance between them is defined to be the rank over $F_{q}$ of the $m \times n$ matrix corresponding 
to $\mathbf{c}-\mathbf{e}$ obtained by expanding each entry of $\mathbf{c}-\mathbf{e}$ as an $m$ tuple along a basis of $F_{q^{m}}$ over $F_{q}$. The rank of $\mathbf{C}$, denoted by $\operatorname{rank}_{q}(\mathbf{C})$ is defined as the minimum of $\operatorname{rank}_{q}(\mathbf{c}-\mathbf{e})$ over all possible pairs of distinct codewords. The rank distance between two codewords $\mathbf{c} \neq \mathbf{e}$ is at most equal to the Hamming distance between them. Combining this with the Singleton bound one gets, $\operatorname{rank}_{q}(\mathbf{C}) \leq \min \{m, n-k+1\}$. The case where $\operatorname{rank}_{q}(\mathbf{C})=n-k+1$ has been studied in [4], [5], and are called Maximum Rank distance (MRD) codes. The rank properties of $(n, k)$ cyclic codes over finite fields $F_{q^{m}},\left(n \mid q^{m}-1,(n, q)=1\right)$ have been studied in [6], [7]. In these, exact expressions and tight bounds for the rank of the code have been derived by making use of the Discrete Fourier Transform (DFT) description of these codes.

In this paper, we derive $m \times n$ STBCs having diversity equal to $n$, suitable for use over block fading channels, from $n$-length cyclic codes over $F_{q^{m}}$ where $n \mid q^{m}-1, m \leq n$ by making use of the rank characterization for cyclic codes [6], [7]. This characterization has been performed by making use of the DFT domain description of cyclic codes [8].

The rest of the content in the paper is organized as follows: In the next section, we will briefly state the theorems associated with the characterization of cyclic codes for the rank metric. In Section III, we obtain $m \times n$ STBCs with diversity equal to $n$ for block-fading channels. In section IV, we present simulation results to show that our codes perform better than the well known codes in terms of codeword error probability and conclude the paper in Section V.

\section{Characterisation OF CyCliC CODES FOR THE RANK METRIC}

We summarize the results relevant to the design of STBCs in Theorems 2.1, 2.2 and 2.3. The proofs of the first two theorems are given in [6], [7] and that of the third is omitted due to space limitations.

Theorem 2.1: Let $\mathbf{C}$ be a cyclic code of length $n \mid q^{m}-$ 1, $(m \leq n)$ over $F_{q^{m}}$ such that the transform component $A_{j q^{s}} \in A_{[j]},|[j]|=e_{j}, e_{j} \mid m$ is free and all other transform components are constrained to zero. Then $\operatorname{rank}_{q}(\mathbf{C})=e_{j}$. In other words, for a length $n \mid q^{m}-1$ cyclic code over $F_{q^{m}}$ with only one transform component non zero, the rank of the code is equal to the size of the $q$-cyclotomic coset to which the free transform component belongs.

In practice, we choose the free transform domain component $A_{j q^{s}} \in A_{[j]}$ where $e_{j}=|[j]|=m$ from a full size $q$ cyclotomic coset. Then from Theorem 2.1 it follows that the rank of the resulting code is $m$. This code has $q^{m}$ codewords. These codes are defined over $F_{q^{m}}$, have length equal to $n$ and dimension equal to 1 .

Theorem 2.2: Let $\mathbf{C}$ be a cyclic code of length $n \mid q^{m}-1$ over $F_{q^{m}}$ whose free transform domain components are $A_{j q^{r}}$ and $A_{j q^{r+s}}$. (the indices of the free transform domain components belong to the same $q$ - cyclotomic coset and $s$ denotes the seperation between them. $\left(1 \leq s \leq e_{j}-1\right),\left(0 \leq r \leq e_{j}-2\right)$. Let all other transform components be constrained to zero. Then, $\operatorname{rank}_{q}(\mathbf{C})=\left(e_{j}-\operatorname{gcd}\left(s, e_{j}\right)\right)$.
This Theorem shows that if we try to increase the number of codewords by considering codes with two free transform components from the same $q$-cyclotomic coset and constraining all other transform components to zero, we can no longer obtain full-rank cyclic codes. Hence, in our search for fullrank STBCs for block-fading channels, we shall confine our study to one dimensional cyclic codes of length $n$ over $F_{q^{m}},\left(n \mid q^{m}-1\right)$.

Theorem 2.3: Let $\mathcal{C}$ be a cyclic code of length $n \mid q^{m}-1$ over $F_{q^{m}}$ such that the transform domain component $A_{j q^{s}} \in A_{[j]}$ is free and all other transform components are constrained to zero. Let $|[j]|=e_{j}$. Consider any non zero codeword $\mathbf{a} \in \mathcal{C}$.

$\mathbf{a}=\left(a_{0}, a_{1}, \cdots a_{e_{j}-1}, \cdots, a_{k e_{j}}, \cdots a_{(k+1) e_{j}-1}, \cdots a_{n-1}\right)$

There are two cases:

(i) $e_{j}$ divides $n$ : If $e_{j} \mid n$, the $\frac{n}{e_{j}}$ sets $\left.\left\{a_{0}, \cdots a_{e_{j}-1},\right\},\left\{a_{e_{j}}, \cdots a_{2 e_{j}-1}\right\} \cdots\left\{a_{n-e_{j}} \cdots a_{n-1}\right)\right\}$

are linearly independent sets over $F_{q}$. If these sets are viewed as $m \times e_{j}$ matrices over $F_{q}$, then each matrix has $F_{q}$-rank equal to $e_{j}$.

(ii) $e_{j}$ does not divide $n$ : If $e_{j}$ does not divide $n$, the $\left\lfloor\frac{n}{e_{j}}\right\rfloor$ sets $\left\{a_{0}, \cdots a_{e_{j}-1}\right\},\left\{a_{e_{j}}, \cdots a_{2 e_{j}-1}\right\}, \cdots$

$\left\{a_{n-\left\lfloor\frac{n}{e_{j}}\right\rfloor-e_{j}} \cdots a_{n-\left\lfloor\frac{n}{e_{j}}\right\rfloor-1}\right\}$ are linearly independent and have rank $e_{j}$ when viewed as $m \times e_{j}$ matrices over $F_{q}$. The last set $\left\{a_{n-\left\lfloor\frac{n}{e_{j}}\right\rfloor} \cdots a_{n-1}\right\}$ consisting of $n-\left\lfloor\frac{n}{e_{j}}\right\rfloor e_{j}$ terms is also linearly independent and has $F_{q}$-rank equal to $n-\left\lfloor\frac{n}{e_{j}}\right\rfloor e_{j}$ when viewed as a $m \times\left(n-\left\lfloor\frac{n}{e_{j}}\right\rfloor e_{j}\right)$ matrix over $F_{q}$.

This theorem is particularly useful in deriving STBCs suitable for block-fading channels from cyclic codes. Also, it follows that a codeword of length $n$ over $F_{q^{m}}$ can be divided into several blocks of length $e_{j}$, each of which can be viewed as a full-rank $m \times e_{j}$ matrix over $F_{q}$. If the length of the fading block is equal to $e_{j}$, it follows from the block-fading sum of ranks criterion, that the effective diversity advantage offered by the code is increased.

We shall use Theorems 2.1 and 2.3 to derive STBCs for block-fading channels from $n$-length cyclic codes over $F_{q^{m}}$.

\section{DESIGNS FOR BLOCK-FADING CHANNELS FROM CYCLIC CODES}

Definition 1: A rate- $k / n, n \times l$ linear design over a field $F \in \mathbb{C}$ is an $n \times l$ matrix with all its entries $F$-linear combinations of $k$ complex variables and their conjugates which are allowed to take values from the field $F$.

Let $(n, q)=1$ and $n \mid q^{m}-1$, where $q$ is either 2 or a prime of the form $4 k+1$. Let $[j]_{n}$ be a $q$-cyclotomic coset of $I_{n}$ of size $m$. By restricting $A_{j}, j \in[j]_{n}$, to $F_{q^{m}}$ and constraining all other transform components to zero, we have a $n$-length cyclic code over $F_{q^{m}}$ whose codewords are of the form,

$$
\left[\begin{array}{lllll}
A_{j}, & \beta^{-j} A_{j}, & \beta^{-2 j} A_{j}, & \cdots & \beta^{-(n-1) j} A_{j}
\end{array}\right]
$$

where $\beta$ is a primitive $n$-th root of unity in $F_{q^{m}}$ and $A_{j} \in F_{q^{m}}$. Viewing $A_{j}$ as a $m$-length column vector over $F_{q}$, the codewords can be viewed as $m \times n$ matrices over $F_{q}$ 
given by,

$$
\left[\begin{array}{ccccc}
a_{0,0} & a_{0,1} & a_{0,2} & \cdots & a_{0, n-1} \\
a_{1,0} & a_{1,1} & a_{1,2} & \cdots & a_{1, n-1} \\
\vdots & \vdots & \vdots & \vdots & \vdots \\
a_{m-1,0} & a_{m-1,1} & a_{m-1,2} & \cdots & a_{m-1, n-1}
\end{array}\right]
$$

where $\beta^{-k j} A_{j}=\sum_{i=0}^{m-1} a_{i, k} \alpha^{i}, a_{i, k} \in F_{q}$ and $\alpha$ is a primitive element of $F_{q^{m}}$. Notice that (6) is a design over $F_{q}$. Also, note that this is in general, possible for any linear code, however, we have information about the rank, only in the case of cyclic codes.

Example 1: Let the number of transmit antennas $N_{t}=2$. We take $n=3$. The 2-cyclotomic coset of 1 modulo 3 is $\{1,2\}$. With $A_{1}$ taking all of $F_{4}$ and other transform components constrained to zero, we have a rank-2 cyclic code $\mathbf{C}_{1}$ over $F_{4}$. Let $\alpha$ be a cube root of unity in $F_{4}$. With $x^{2}+x+1$ as the minimal polynomial of $\alpha$, the codewords of $\mathbf{C}$ are of the form,

$$
\left[\begin{array}{ccc}
a_{0} & a_{0}+a_{1} & a_{1} \\
a_{1} & a_{0} & a_{0}+a_{1}
\end{array}\right]
$$

where $a_{0}, a_{1} \in F_{2}$.

This is an example of a rate $2 / 3$ design. A design based on a cyclic code of length $n$ over $F_{q^{m}}$ is used over a blockfading channel where the channel is known to remain invariant over $m$ successive signalling intervals. These $m$ successive signalling intervals constitute a block. Therefore, we have two cases.

(i) $m$ divides $n$ : In this case, each codeword encompasses an integral number of fading blocks. As these codes, can be decomposed into $\frac{n}{m}$ blocks, the components of each of which are linearly independent, we have a $m \times n$ matrix over $F_{q}$, which can be decomposed into $\frac{n}{m}$ submatrices, each of $F_{q^{-}}$ rank $m$.

(ii) $m$ does not divide $n:$ In this case, we can either delete $\left(n-\left\lfloor\frac{n}{m}\right\rfloor m\right)$ columns or add $m-\left(n-\left\lfloor\frac{n}{m}\right\rfloor m\right)$ columns such that the last block also consists of linearly independent elements. In the first case, we gain in code rate at the expense of diversity and in the second case we loose in rate and gain in diversity.

To obtain STBCs from the above designs, we have to map the elements of $F_{q}$ into the complex field such that the full-rank property of the finite field design is preserved. We call a signal set, which is a finite subset of the complex field, as a signal set matched to $F_{q}$, if there exists a map from $F_{q}$ to the signal set which is an isometry for the $F_{q}$-rank to the complex field rank. There are two methods for obtaining maps, the Hammons and El Gamal map [9] (suitable for codes over extension fields of $F_{2}$ ) and the map proposed by Lusina, Gabidulin and Bossert [10].

An $n$-length cyclic code over $F_{2^{m}}$ will give rise to an $m \times n$ STBC with $2^{m}$ codewords for $m$ transmit antennas. Hence, the code rate in bits per channel use is $\frac{1}{n} \log _{2}\left(2^{m}\right)=m / n$. Now, assuming that we want full-rank STBCs, we have the condition $m \leq n$. Therefore, for the case of cyclic codes over $F_{2^{m}}$, the data rate is always upper bounded by 1 bit per channel use and we will not consider the Hammons and El Gamal map here. To achieve higher code rates, we derive STBCs from non binary cyclic codes by making use of the Lusina et al. map.

\section{A. $q=4 k+1$, Lusina, Gabidulin and Bossert[10]}

Let $q$ be a prime of the form $q=4 k+1$. By definition a Gaussian integer $w$ is a complex number defined as $w=$ $a+i b, a, b \in \mathbf{Z}, i=\sqrt{-1}$. From number theory, it is known that every prime number $q$ of the form $q \equiv 1 \bmod 4$ can be written as $q=(u+i v) \times(u-i v)=u^{2}-v^{2}$. The number $\Pi=u+i v$ is known as Gaussian prime number where $u, v \in \mathbf{Z}$. Let $\Pi^{\prime}=u-i v$. Then calculation modulo $\Pi$ is defined as, $\zeta=w$ modulo $\Pi=w-\left[\frac{w \Pi^{\prime}}{\Pi \Pi^{\prime}}\right] \Pi$ where [.] performs the operation of rounding to the nearest Gaussian integer. The Gaussian integers modulo $\Pi$ form a field, $G_{\pi}=$ $\left\{\zeta_{0}=0, \zeta_{1}=1, \zeta_{2}, \cdots, \zeta_{q-1}\right\}$ and the map $\xi: F_{q} \Rightarrow G_{\Pi}$ given by $\zeta_{i}=i \bmod \Pi=i-\left[\frac{i \Pi^{\prime}}{\Pi \Pi^{\prime}}\right] \Pi, i=0,1,2, \cdots, p-1$ is an isomorphism [10]. Therefore when we map codewords from a linear cyclic code over $F_{q^{m}}, q=5,13,17, \cdots$ which are $m \times n$ matrices over $F_{q}$ to $m \times n$ matrices over the complex Gaussian field, the full-rank property of the code over $F_{q^{m}}$ is preserved. We give below, an example of the map between $F_{5}$ and the corresponding complex Gaussian field.

Example 2: The map between $F_{5}$ and $G_{\Pi}$ where $\Pi=1+i 2$ is defined by,

$0 \mapsto 0,1 \mapsto 1,2 \mapsto 0+i 1,3 \mapsto 0-i 1,4 \mapsto-1$.

Example 3: Let $N_{t}=2$ and $q=5$. Let the length of the cyclic code be $n=6$. The 5 -cyclotomic coset of $1 \bmod 6$ is $\{1,5\}$. With $A_{1}$ taking all of $F_{25}=F[x] /\left(x^{2}+x+1\right)$ and all other transform components constrained to zero, we have a rank- 2 cyclic code over $F_{5}$ with 25 codewords which can be expressed as $2 \times 6$ matrices over $F_{5}$. We make use of the map proposed by Lusina et al. to derive a full-rank $2 \times 6$ STBC. From the block-fading sum of ranks criteria, the diversity of this code is 6 . The codewords of the STBC are of the form given in (8), where $a_{0}, a_{1} \in F_{5} . \xi: F_{5} \mapsto G_{1+2 i}$. The code rate of this code in bits per channel use is, $\frac{\log _{2}(25)}{6}=0.774$.

Example 4: Let $N_{t}=2$ and $q=13$. Let the length of the cyclic code be $n=7$. The 13 -cyclotomic coset of $1 \bmod 13$ is $\{1,6\}$. With $A_{1}$ taking all of $F_{169}$ generated by $F[x] /\left(x^{2}+\right.$ $x+2)$ and all other transform components constrained to zero, we have a rank- 2 cyclic code over $F_{13}$ with 169 codewords which can be expressed as $2 \times 7$ matrices over $F_{13}$. To increase the rate of STBC derived from this cyclic code, we will delete the last column and then map the resulting $2 \times 6$ matrices over $F_{13}$ using the map $\xi: F_{13} \mapsto G_{2+3 i}$. This yeilds a full-rank $2 \times 6$ STBC with 169 codewords. The diversity of this code is 6 . The codewords of this code are of the form given in (9). The code rate in bits per channel use is $\frac{\log _{2}(169)}{6}=1.233$.

Example 5: Let $N_{t}=2$ and $q=17$. Let the length of the cyclic code be $n=6$. The 17 -cyclotomic coset of $1 \bmod 6$ is $\{1,5\}$. With $A_{1}$ taking all of $F_{289}=F[x] /\left(x^{2}+x+3\right)$ and all other transform components constrained to zero, we have a rank-2 cyclic code over $F_{17}$ with 289 codewords which can 


$$
\begin{gathered}
{\left[\begin{array}{llllll}
\xi\left(a_{0}\right) & \xi\left(4 a_{0}+a_{1}\right) & \xi\left(3 a_{0}+a_{1}\right) & \xi\left(4 a_{0}\right) & \xi\left(a_{0}+4 a_{1}\right) & \xi\left(2 a_{0}+4 a_{1}\right) \\
\xi\left(a_{1}\right) & \xi\left(2 a_{0}+2 a_{1}\right) & \xi\left(2 a_{0}+a_{1}\right) & \xi\left(4 a_{1}\right) & \xi\left(3 a_{0}+3 a_{1}\right) & \xi\left(3 a_{0}+4 a_{1}\right)
\end{array}\right]} \\
{\left[\begin{array}{llllll}
\xi\left(a_{0}\right) & \xi\left(3 a_{0}+5 a_{1}\right) & \xi\left(4 a_{0}+11 a_{1}\right) & \xi\left(7 a_{0}+a_{1}\right) & \xi\left(a_{0}+12 a_{1}\right) & \xi\left(3 a_{0}+2 a_{1}\right) \\
\xi\left(a_{1}\right) & \xi\left(9 a_{0}+3 a_{1}\right) & \xi\left(a_{0}+3 a_{1}\right) & \xi\left(6 a_{0}+a_{1}\right) & \xi\left(7 a_{0}+7 a_{1}\right) & \xi\left(12 a_{0}+4 a_{1}\right)
\end{array}\right]} \\
{\left[\begin{array}{llllll}
\xi\left(a_{0}\right) & \xi\left(15 a_{0}+12 a_{1}\right) & \xi\left(14 a_{0}+8 a_{1}\right) & \xi\left(16 a_{0}+5 a_{1}\right) & \xi\left(2 a_{0}+a_{1}\right) & \xi\left(3 a_{0}+2 a_{1}\right) \\
\xi\left(a_{1}\right) & \xi\left(12 a_{0}+7 a_{1}\right) & \xi\left(12 a_{0}+7 a_{1}\right) & \xi\left(3 a_{1}\right) & \xi\left(5 a_{0}+a_{1}\right) & \xi\left(5 a_{0}+4 a_{1}\right)
\end{array}\right]}
\end{gathered}
$$

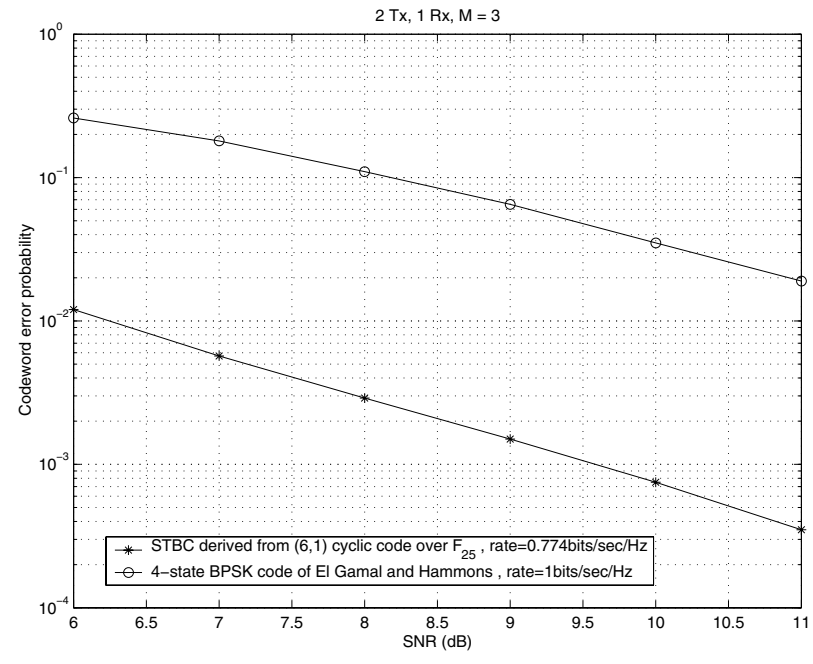

Fig. 1. Performance comparison of length 6 full-rank STBC with diversity of 6 derived from $(6,1)$ cyclic code over $F_{5^{2}}$ with the best 4 state BPSK space time code derived by El Gamal and Hammons.

be expressed as $2 \times 6$ matrices over $F_{17}$. We make use of the map $\xi: F_{17} \mapsto G_{4+i}$ to derive a full-rank $2 \times 6$ STBC. From the block-fading sum of ranks criteria, we conclude that the diversity of this code is 6 . The code rate in bits per channel use is $\frac{\log _{2}(289)}{6}=1.3626$. The codewords of this code are as in (10). We can increase the rate of this code by deleting the last two columns. By doing this we obtain a code with diversity of 4. The code rate in bits per channel use is $\frac{\log _{2}(289)}{4}=2.043$.

\section{Simulation Results}

In this section, we present simulation results and compare the performance of our codes with some well known codes for block-fading channels. These include the codes proposed by El Gamal and Hammons [1], Tarokh, Sheshadri and Calderbank [11] and the Turbo Space Time Code proposed by Stefanov and Duman [3].

In Figure 1, we have compared the error performance of our length 6 , full-rank STBC with diversity 6 , rate 0.774 derived from a $(6,1)$ cyclic code over $F_{5^{2}}$ (Example 3 ), with the 4state BPSK Space Time Trellis code (STTC) derived by El Gamal and Hammons [1] which has a rate of 1. This happens to be the best performing El Gamal and Hammons code with rate $1 \mathrm{bit} / \mathrm{sec} / \mathrm{Hz}$. This is the four state $(5,7)$ optimal free distance space time trellis code with diversity equal to 4 . The

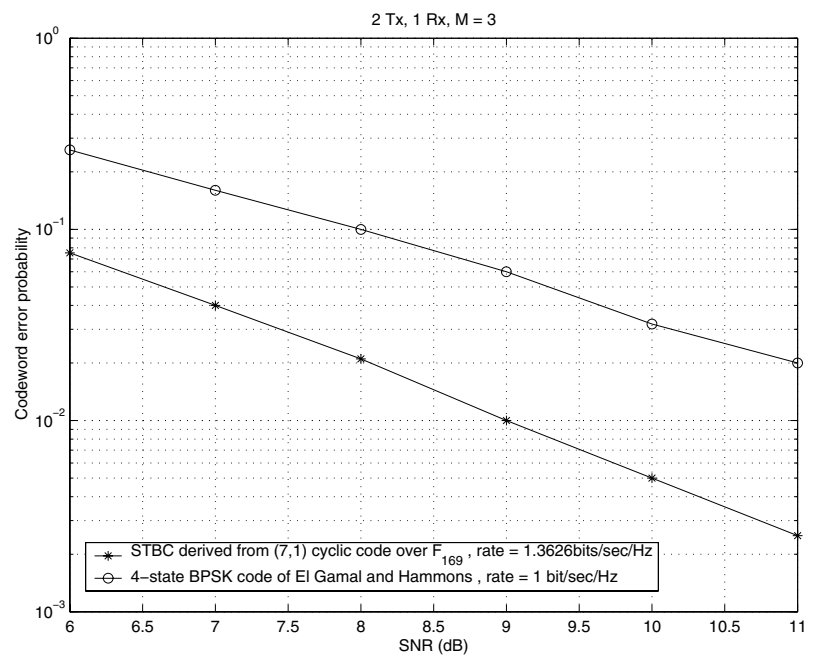

Fig. 2. Performance comparison of length 6 full-rank STBC with diversity of 6 derived from $(7,1)$ cyclic code over $F_{13^{2}}$ with the best 4 state BPSK space time trellis code derived by El Gamal and Hammons.

parameters of this code are, $N_{t}=2, N_{r}=1$, number of fading blocks per codeword $M=3$, rate $=1$. Our code also has the same operational parameters but has rate equal to .774 . While the rate of our code is less by about 0.226 , we observe that we are able to obtain any given probability of error with a much reduced value of SNR (at least $5 \mathrm{~dB}$ less).

In Figure 2, we have compared the error performance of our length 6 , full-rank STBC with diversity 6 , rate 1.233 derived from a $(7,1)$ cyclic code over $F_{13^{2}}$ (Example 4$)$, with the 4state BPSK STTC derived by El Gamal and Hammons. This is the same El Gamal and Hammons code referred to in the context of Figure 1. We observe that our code outperforms this code in both rate and in error performance. (This code is able to achieve any given probability of error at a SNR which is at least $2.5 \mathrm{~dB}$ less than that required by the El Gamal and Hammons code). The number of blocks, $M=3$ for both the codes.

In Figure 3, we have compared the error performance of our length 4, full-rank STBC with diversity 4, rate 2.043 derived from a $(6,1)$ cyclic code over $F_{17^{2}}$ (Example 5), with the following:

- The linear $\mathbf{Z}_{4}$ code obtained by lifting the binary $(6,7)$ code with QPSK modulation [1] with parameters, $N_{t}=2, M=2$, rate $=2 b i t s / \mathrm{sec} / \mathrm{Hz}$. 


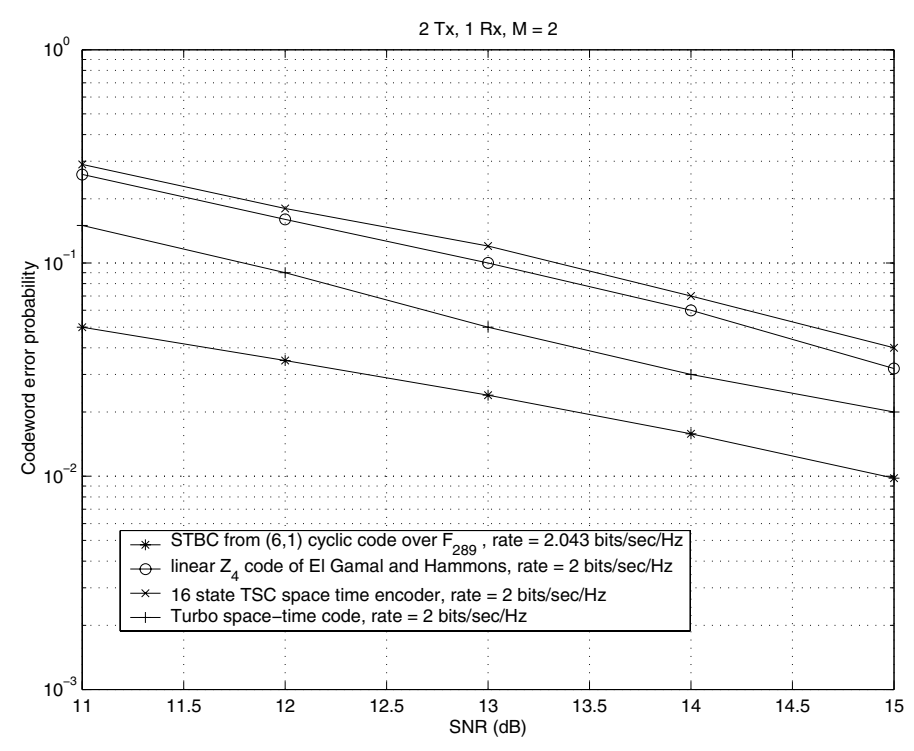

Fig. 3. Performance comparison of length 4 full-rank STBC with diversity of 4 derived from $(6,1)$ cyclic code over $F_{17^{2}}$ with the best 16 state QPSK space time code derived by El Gamal and Hammons.

- The 16 state code derived by Tarokh, Sheshadri and Calderbank (TSC) code [11] with rate 2 bits/sec/Hz.

- New Turbo Space Time code [3] with interleaver size $N=260$, rate $=2 b i t s / \sec / \mathrm{Hz}$. The path gains are assumed to be constant for a period of 65 transmissions. We observe that our code outperforms all of the above in rate as well as error performance.

In Figure 4, we compare the error performance of our length 6 full-rank STBC with diversity 6 , rate 1.3626 derived from $(6,1)$ cyclic code over $F_{17^{2}}$ (Example 5), with the 4 state BPSK space time trellis code proposed by El Gamal and Hammons which has a rate of 1 . This is the same El Gamal and Hammons code referred to in the context of the Figure 1. The number of blocks $M=3$ for both the codes. We observe that our codes outperform the codes proposed by El Gamal and Hammons in both rate and error performance.

\section{Discussion}

We have shown that it is possible to obtain designs for full-rank STBCs matched to MIMO block-fading channels from length $n \mid q^{m}-1$ cyclic codes over $F_{q^{m}}$. We have derived performance curves (codeword probability of error as a function of signal to noise ratio) for some of these codes. From the simulation results, we see that these codes offer superior performance as compared to the codes derived in [1], [11] and the Turbo Space time code proposed in [3].

\section{REFERENCES}

[1] H. El Gamal and A. R Hammons, Jr., "On the Design of Algebraic Space-Time Codes for MIMO Block-Fading Channels ,"IEEE Trans. on

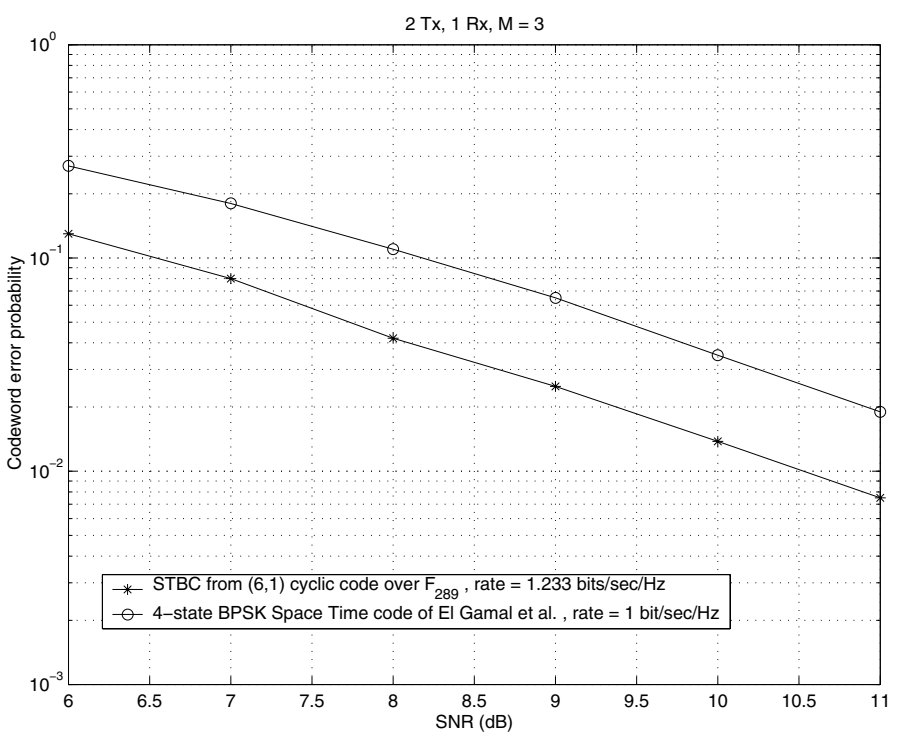

Fig. 4. Performance comparison of length 6 full-rank STBC with diversity of 6 derived from $(6,1)$ cyclic code over $F_{17^{2}}$ with the best 4 state BPSK space time code derived by El Gamal and Hammons.

Inform. Theory, vol.49, No.1, Jan.-2003, pp. 151-163.

[2] E. Biglieri, G. Caire and G. Taricco, "Limiting performance of block-fading channels with multiple antennas," IEEE Trans. Inform. Theory, vol.47, no.4, May 2001, pp.1273 - 1289

[3] A. Stefanov and T. Duman,"Turbo coded modulation for systems with transmit and receive diversity over Block-fading channels: System model, decoding approaches and practical considerations," IEEE J. Select Areas Commun., vol. 19, pp 958-968, May 2001.

[4] E. M. Gabidulin, "Theory of Codes with Maximum Rank Distance," Problemy Peredachy Informatsii, 21, 99.3-14, Jan.Mar. 1985.

[5] R. M. Roth, "Maximum Rank Array Codes and their application to Criss Cross Error Correction," IEEE Trans. Inform. Theory, vol.37, pp.328-336, March 1991.

[6] U. Sripati and B. Sundar Rajan, " On the Rank Distance of Cyclic Codes," Proc. IEEE Int. Symp. Inform. Theory, Yokohoma, Japan, June-July 2003, p.72.

[7] B. Sundar Rajan and U. Sripati, "On the Rank Distance of Cyclic Codes,” Technical Report no. TR-PME-2003-04 Department of Electrical Communication Engineering, Indian Institute of Science, Bangalore-560012. Available for download at http://ece.iisc.ernet.in/ bsrajan.

[8] R. E. Blahut, Theory and Practise of Error Control Codes, Addison Wesley, 1983.

[9] A. Roger Hammons Jr. and Hesham El Gamal, "On the Theory of Space Time Codes for PSK modulation," IEEE Trans. on Inform. Theory, vol.46, No.2, pp.524-542, March-2000.

[10] Paul Lusina, E. Gabidulin and Martin Bossert, "Maximum Rank Distance Codes as Space Time Codes," IEEE Trans. on Inform. Theory, vol.49, No.10, Oct. 2003, pp. 2757-2760.

[11] V. Tarokh, N. Seshadri and A. R. Calderbank, "Space-time codes for high data rate wireless communication: Performance criterion and code Construction," IEEE Trans. Inform. Theory, vol. 44, pp.744-765, Mar-1998. 